\title{
Stem Cell Transplantation for Traumatic Spinal Cord Injury: What Have We Learned from Previous Experience
}

\author{
Elhawary S., Wagih A., Essam B., Tarek I., Aamer M., Ellessy R. M. \\ Faculty of Medicine, Cairo University, Cairo, Egypt
}

Email address:

halagabr@yahoo.com

To cite this article:

Elhawary S., Wagih A., Essam B., Tarek I., Aamer M., Ellessy R. M.. Stem Cell Transplantation for Traumatic Spinal Cord Injury: What Have We Learned from Previous Experience. American Journal of Bioscience and Bioengineering. Special Issue: Stem Cells for Neuroregeneration: Where Do We Stand. Vol. 3, No. 4-1, 2015, pp. 34-42. doi: 10.11648/j.bio.s.2015030401.16

\begin{abstract}
Spinal cord injuries represent one of the most devastating illnesses that can affect the human body. Before the advent of the regenerative medicine era, it was regarded as an untreatable condition. Stem cell plasticity and translational medicine research open a new window of hope for this category of patients. Clinical trials of stem cell therapy for spinal cord injuries are now more than a decade old. However, the diversity of clinical trial design, cell type, dose and route of injection, make it extremely difficult to draw decisions from these previous experience. This review tries to collect as much evidence as we can from previous studies in order to suggest paths for future research in this setting.
\end{abstract}

Keywords: Stem Cell Therapy, Spinal Cord Injury, Mesenchymal Stem Cells, Regenerative Medicine, Olfactory Ensheathing Cells

\section{Introduction}

Spinal cord injury (SCI) is one of the most disabling acquired medical conditions affecting thousands of individuals per year. Furlan and coworkers reported a global incidence of SCI of 8.0 to 246.0 cases per million per year(1) The medical, social and psychological sequelae of SCI are enormous, especially that the current medical and supportive management have little impact on the overall quality of life.

Stem cell therapy is an emerging field of medicine which holds great promise for regenerating tissues and organs. The application of stem cell therapy for SCI is a dream that tackled minds of researchers for years now. After more than a decade of stem cell research for neuroregeneration of SCI, we need to put together evidence we gained and re-evaluate future prospectives in this area.

\section{Stem Cells}

Stem cells are self-renewing unspecialized cells with variable degrees of plasticity (2). Since first identified by Till and McCulloch in 1963, identification of stem cell characteristics has been a point of vast research (3). Stem cells have the ability to proliferate and differentiate. In addition, they possess the very important characteristic of homing to their place of origin when injected systemically.

Stem cells can be isolated from nearly all body tissues. According to their origin, stem cells are classified into hemopoeitic stem cells (HSCs), mesenchymal stem cells (MSCs), adipose derived stem cells (ADSCs), cord blood stem cells, and organ-specific stem cells (4).

Stem cell transplantation for hemopoeitic disorders came into practice since 1968 (5). Observations following extrahematologic regenerative action of injected stem cells in bone marrow transplantation coined the biologic phenomenon of "plasticity" in which stem cells cross lineage boundaries and adopt biologic characteristics of nonhematologic lineages(6). These observations laid the foundations of "regenerative medicine" (7).

Can Stem Cells Differentiate into Neural Lineage?

MSCs, whether bone marrow derived or adipose derived have been shown to differentiate into neuron cells (8-10), Schwann Cells (11-13), neurotrophic factor secreting cells (14-15), and glial cells (16). Researchers have been able to produce mature and functional dopaminergic neurons from induced pleuripotent stem cells (iPS) $(17,18)$ and Wharton jelly derived MSCs (19). Induction of motor neuron differentiation from embryonic stem cell was successful in many laboratories (20). Various stem cell types have been directed to differentiate into all neural cells such as motor neurons, dopaminergic neurons, oligodendrocytes, astrocytes 
and microglia (21).

Possible Mechanisms of Stem cell-induced Neurogeneration:

Vast research, both in-vitro and animal experiments, tried to elucidate the mechanism of neuroregenerative action of stem cells. A number of mechanisms have been proposed: transplanted MSCs induce endogenous neural growth (22). MSC modify micorenvironment to produce a more inductive one for regeneration. This is through decreasing apoptosis associated with injury or inflammation. Moreover, MSCs modulated inflammatory response through (23).

Actual transdifferentiation of stem cells into neural lineage, although not the main pathway of regeneration, is another mechanism $(24,25)$.

Other mechanisms exclusively found with MSCs are immunomodulation $(26,27)$ gene delivery (28-30) and angiogenesis (31-33).

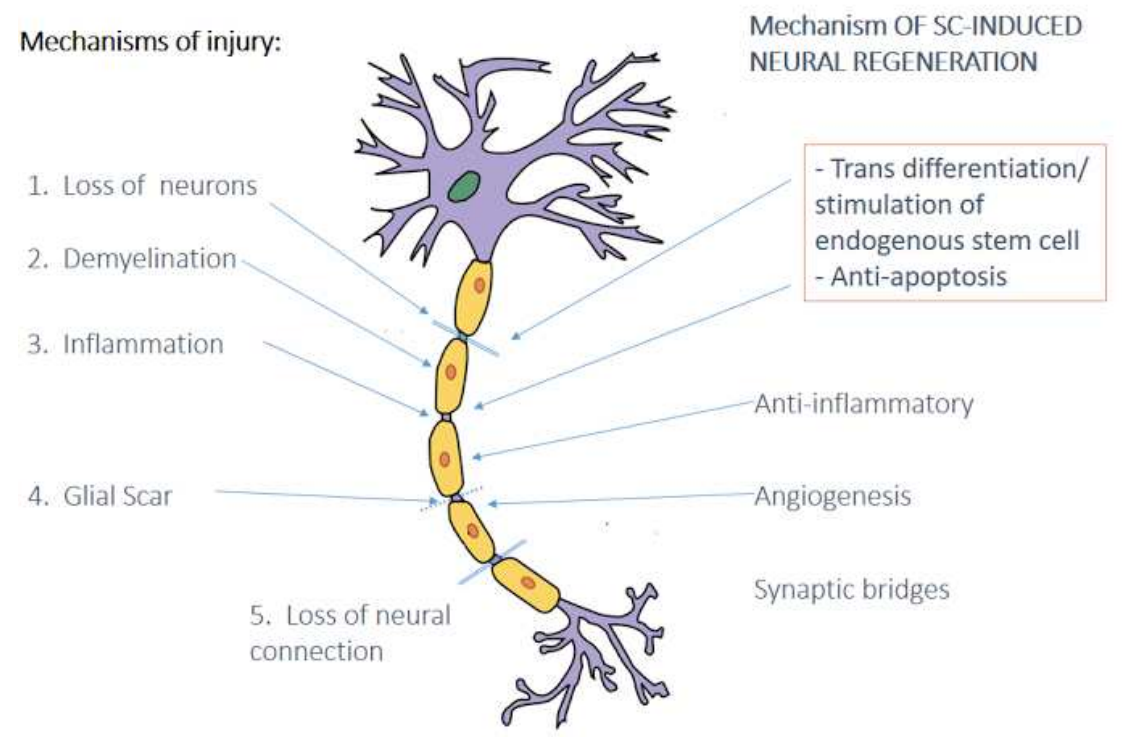

Figure (1). Possible mechanisms of Stem cell-mediated neural recovery after traumatic spinal cord injury.

\section{Stem Cell Clinical Trials}

Methodology of Research: On searching the website:"www.clinicaltrials.gov" on 19 May, 2015 using the search words "stem cell therapy", we found 4496 studies. When we narrowed the search to "stem cell therapy for spinal cord injuries" we got 33 studies. $6(18.2 \%)$ of them were completed, one $(3 \%)$ terminated, two $(6 \%)$ suspended and the other $(72.7 \%)$ are still recruiting. Search for published clinical trials on pubmed was done using the search words : "stem cells/spinal cord injury" with filters :"clinical trials/human". On google scholar, the search words used were:"stem cells, spinal cord injury AND clinical trials AND human. Eighteen trials were found, three using olfactory ensheathing cells while the others used bone marrow derived stem cells.

The first published trials found dealt with olfactory ensheathing cells : three trials were found published in 2005 , 2006, and 2008 .

On 2005, Feron et al (34) published a phase I, controlled single-blinded study enrolling 6 patients: 3 serving as cases and 3 serving as control. Olfactory ensheathing cells were obtained through transnasal endoscopic excision from the nasal septum. Cells were then injected into the spinal cord under microscopic control. Dose escalation was done injecting 12, 24 and 48 million cells. Evaluation was done using radiological assessment for evidence of local complications or tumors, ASIA scale for motor and sensory improvement, mental health assessment, clinical outcome variables (COV) scale for respiratory functions and clinical examination. The authors reported that the procedure was safe and well-tolerated with no reported complications. However, they reported minimal improvement in light touch and pinprick scores but are below clinical detectable levels; with no motor improvement.

In 2006, Lima et al (35) published his trial using olfactory ensheathing cells but grafting them in SCI site surgically, in a pilot safety trial enrolling 7 patients. The authors evaluated improvement using : ASIA score, conventional electromyography, full spinal cord MRI scan, otolaryngological evaluation, CT scan of the nose and paranasal sinuses, psychological assessment, in addition to pre and post-operative urodynamic studies.

The authors reported improvement in ASIA motor scores in all patients, sensory scores in 6 patients and urodynamic studies in 2 patients. They reported that procedure was safe and well-tolerated and recommended further controlled trials.

In 2007, two trials using bone marrow derived cells were published. Yoon and co-workers (36) published a phase I/II controlled, open-label non-randomized trial enrolling 37 patients and 12 controls. The workers used bone- marrow derived mononuclear cell population which includes stem 
cells in a dose of $2 \times 10^{8}$ cells after prior stimulation using G-CSF. Cells were injected locally under microscopic control. The study used ASIA scale and motor evoked potential in addition to MRI for follow-up. Mild complications in the form of fever, neuropathic pain and abdominal pain were reported. The efficacy reported was improved ASIA scores in acute and subacute, but not in chronic SCI patients.

Chernykh et al (37) published a phase I controlled trial exploring the safety and efficacy of bone marrow mononuclear cell injection, both locally into the cystic cavity of the spinal cord and intravenously in a dose of $10.9 \times 10^{6}$. The study evaluated the efficacy using ASIA, Bartel and Ashworth scales. No complications were reported. 12 patients showed sensory improvement, 4 patients showed improvement in motor functions and conduction and 2 patients showed improvement in bladder control.

In 2009, Cristante and colleagues (38) published a prospective non-randomized, non-controlled clinical trial enrolling 39 patients. The study used mobilized bone marrow stem cells collected from the peripheral blood in a dose of 2.5 X $10^{6}$ leukapharesis-derived mononuclear cells. Patients were followed using somatosensory evoked potential. The results showed positive response in SSEP in 26 patients.

In 2010, three clinical trials were published:

First report of MSC, was in the work of Abo ElKheir et al (39), in 2010 which compared between BM and PB derived MSCs in cases of chronic SCI. The study recruited 150 chronic SCI patients, randomly divided into bone marrow aspiration, stem cell mobilization using G-CSF and control group. Bone marrow group received MSCs in a dose of 2 $\mathrm{X} 10^{6} / \mathrm{kg}$ in 6 monthly divided doses. Peripheral blood patients received 5 doses of G-CSF and after leukapharesis, received MSCs in the same dose . Patients were followed using ASIA score, trunk motor score and sphincteric control questionnaire. The research team reported a statistically significant mild increase in ASIA score and trunk motor score in patients as compared to controls. Bone marrow group showed slightly more improvement in the motor scores, although not statistically significant. The study reported mild self-limited complications.

Abdelaziz et al (40), in 2010 reported a controlled trial enrolling 30 chronic SCI patients divided as 20 cases and 10 control. The study used autologous bone marrow derived MSCs in a dose of $5 \times 10^{6} / \mathrm{kg}$. Cells were delivered intraparenchymal and intralesional through open surgery. Two subsequent intrathecal injections were given in two consecutive months. Follow-up was done using clinical assessment using ASIA IMSOP scales, electrophysiology and MRI. The trial reports $30 \%$ improvement in ASIA scale A patients, $60 \%$ improvement in ASIA scale B patients, with no motor improvement in ASIA scale C patients. Sensory and sphincteric improvement was seen in 7 out of the 20 patients with no correlation to original ASIA scale. The workers reported safety of the procedure with no complications developing on follow-up.

Kishk et al (41) described the results of a case-control series involving chronic SCI patients. The study enrolled 44 patients and 20 controls. Autologous MSCs were injected intrathecally in a dose of $5-10 \times 10^{6} / \mathrm{kg}$ in six divided doses. Patients were followed using ASIA impairment scale, ASIA grading for completeness of injury, Ashworth Spasticity scale, Functional ambulation classification and bladder and bowel control questionnaire. The study reported the improvement in ASIA score and Grade. However, the workers reported complications such as neuropathic pain, increased spasticity and one case who developed encephalomyelitis.

Abo Elkheir et al (42), in 2013 published the result of a registered phase I/II controlled single-blind clinical trial (clinicaltrials.gov identifier: NCT00816803). The study enrolled 70 chronic cervical and thoracic SCI patients with injury durations of at least 6 months were treated with either intrathecal injection of autologous adherent bone marrow cells combined with physical therapy, or with physical therapy alone. Patients were evaluated with ASIA score, electrophysiological somatosensory evoked potential, MRI imaging, and functional independence measurements. The study reported that chronic cervical and thoracic SCI patients treated with autologous adherent bone marrow cells combined with physical therapy showed functional improvements over patients in the control group treated with physical therapy alone, and there were no cell therapy-related side effects. At 18 months post-treatment, 23 of the 50 cell therapy-treated cases (46 percent) showed sustained improvement using the American Spinal Injury Association (ASIA) Impairment Scale (AIS). Compared to those patients with cervical injuries, a higher rate of functional improvement was achieved in thoracic SCI patients with shorter durations of injury and smaller cord lesions.

Table (1). Studies using Stem Cell Transplantation for treatment of Spinal Cord Injuries.

\begin{tabular}{|c|c|c|c|c|c|c|}
\hline Investigators & Study Design & Cell dose \& Type & Mode Of Delivery & Patient diagnosis & Evaluation Scales & Main Results \\
\hline $\begin{array}{l}\text { Elkheir et al, } \\
2013(42)\end{array}$ & $\begin{array}{l}\text { I/II } \\
\text { Controlled } \\
\text { single blind } \\
\text { Sample size: } \\
70 \text { patients }\end{array}$ & $\begin{array}{l}\text { Bone marrow derived } \\
\text { MSCs }\end{array}$ & Intrathecal & $\begin{array}{l}\text { Chronic spinal } \\
\text { cord injury }\end{array}$ & $\begin{array}{l}\text { ASIA score } \\
\text { SSEP } \\
\text { MRI } \\
\text { Functional } \\
\text { independence } \\
\text { measure }\end{array}$ & $\begin{array}{l}\text { Improvement in } \\
46 \%\end{array}$ \\
\hline $\begin{array}{l}\text { Dai et al , } 2013 \\
\text { (43) }\end{array}$ & $\begin{array}{l}\text { Controlled } \\
\text { Clinical trial } \\
\text { Sample size: } \\
20 \text { study group }\end{array}$ & $\begin{array}{l}\text { BM MSCs } \\
2 \times 107\end{array}$ & $\begin{array}{l}\text { slowly injected to a depth } \\
\text { of } 3 \mathrm{~mm} \text { at multiple sites } \\
\text { in the central dorsal area } \\
\text { across the junction of }\end{array}$ & $\begin{array}{l}\text { Complete and } \\
\text { chronic SCI }\end{array}$ & $\begin{array}{l}\text { AIS grading, ASIA } \\
\text { score }\end{array}$ & $\begin{array}{l}\text { Improvement in } \\
\text { AIS grade } \\
\text { And ASIA } \\
\text { score in } 45 \%\end{array}$ \\
\hline
\end{tabular}




\begin{tabular}{|c|c|c|c|c|c|c|}
\hline Investigators & Study Design & Cell dose \&Type & Mode Of Delivery & Patient diagnosis & Evaluation Scales & Main Results \\
\hline & $\begin{array}{l}20 \text { control } \\
\text { group }\end{array}$ & & $\begin{array}{l}\text { injured and normal spinal } \\
\text { cord. }\end{array}$ & & & Of patients \\
\hline $\begin{array}{l}\text { Karamouzian et } \\
\text { al , } 2012 \text { (44) }\end{array}$ & $\begin{array}{l}\text { Non } \\
\text { randomized } \\
\text { clinical trial } \\
\text { phase I/II } \\
\text { Sample size: } \\
11 \text { study } \\
20 \text { control }\end{array}$ & $\begin{array}{l}7 \times 105 \text { to } 1.2 \times 106 \mathrm{v} \\
\text { Bone marrow } \\
\text { mononuclear cells }\end{array}$ & Intrathecal & $\begin{array}{l}\text { Fracture } \\
\text { dislocation } 54.5 \% \\
\text { Burst fracture } \\
45.5 \%\end{array}$ & ASIA scale & $\begin{array}{l}\text { A two-grade } \\
\text { Improvement in } \\
\text { ASIA scale In } \\
45.5 \% \text { of } \\
\text { patients }\end{array}$ \\
\hline $\begin{array}{l}\text { Park et al, } 2012 \\
(45)\end{array}$ & $\begin{array}{l}\text { Prospective } \\
\text { non-controlled } \\
\text { Sample size: } \\
10 \text { patients }\end{array}$ & $\begin{array}{l}\text { MSCs expanded for } 4 \\
\text { weeks } \\
\text { Dose: } \\
8 \text { X106 direct injection } \\
\text { into the cord } \\
4 \text { X } 107 \text { into intradural } \\
\text { space } \\
\text { Intrathecal injection of } \\
5 \text { X } 107 \text { after } 4 \text { and } 8 \\
\text { weeks }\end{array}$ & $\begin{array}{l}\text { Direct injection in the } \\
\text { cord } \\
\text { Injection in } \\
\text { Intradural space } \\
\text { Intrathecal injection }\end{array}$ & $\begin{array}{l}\text { Chronic spinal } \\
\text { cord injury }\end{array}$ & $\begin{array}{l}\text { ASIA scale } \\
\text { MRI } \\
\text { Electrophysiology }\end{array}$ & $\begin{array}{l}\text { Motor } \\
\text { improvement } \\
\text { In } 6 \text { patients }\end{array}$ \\
\hline $\begin{array}{l}\text { Gabr et al, } 2011 \\
(46)\end{array}$ & $\begin{array}{l}\text { Prospective } \\
\text { non-controlled } \\
\text { trial } \\
\text { Sample size: } \\
419 \text { patients: } 87 \\
\text { cervical, } 323 \\
\text { thoracic, } 9 \\
\text { lumbar }\end{array}$ & $\begin{array}{l}\text { BM-MSCs } \\
2 \text { X } 106 / \mathrm{kg} \\
\text { On } 6 \text { divided monthly } \\
\text { doses }\end{array}$ & Intrathecal & $\begin{array}{l}\text { Chronic spinal } \\
\text { cord injury }\end{array}$ & $\begin{array}{l}\text { ASIA score } \\
\text { Trunk motor score } \\
\text { Bowel and Bladder } \\
\text { score } \\
\text { MRI }\end{array}$ & $\begin{array}{l}43 \% \\
\text { improvement }\end{array}$ \\
\hline $\begin{array}{l}\text { Kishk et al, } \\
2010(41)\end{array}$ & $\begin{array}{l}\text { Case-control } \\
\text { series } \\
\text { Sample size: } \\
44 \text { patients } \\
20 \text { control }\end{array}$ & $\begin{array}{l}\text { BM-MSCs } \\
5-10 \text { X106 }\end{array}$ & Intrathecal & $\begin{array}{l}\text { Chronic spinal } \\
\text { cord injury }\end{array}$ & $\begin{array}{l}\text { ASIA scale } \\
\text { ASIA score } \\
\text { Ashworth scale }\end{array}$ & $\begin{array}{l}\text { Improvement in } \\
\text { ASIA score and } \\
\text { scale }\end{array}$ \\
\hline $\begin{array}{l}\text { Elkheir et al , } \\
2010 \text { (39) }\end{array}$ & $\begin{array}{l}\text { Prospective, } \\
\text { non-controlled } \\
\text { clinical trial } \\
\text { Sample size: } \\
419\end{array}$ & $\begin{array}{l}\text { BM-MSCs } \\
\text { PB-MSCs } \\
2 \text { X106/kg }\end{array}$ & Intrathecal & $\begin{array}{l}\text { Chronic spinal } \\
\text { cord injury: } \\
87 \text { cervical } \\
323 \text { thoracic } \\
9 \text { lumbar }\end{array}$ & $\begin{array}{l}\text { ASIA score } \\
\text { Ashworth scale } \\
\text { Trunk motor score } \\
\text { SSEP } \\
\text { MRI }\end{array}$ & $\begin{array}{l}\text { Improvement: } \\
43.2 \% \text { ovrall } \\
\text { improvement } \\
40.2 \% \text { sensory } \\
16 \% \text { motor } \\
8.6 \% \\
\text { sphincteric }\end{array}$ \\
\hline $\begin{array}{l}\text { Abdelaziz et al, } \\
2010(40)\end{array}$ & $\begin{array}{l}\text { Controlled } \\
\text { prospective } \\
\text { Sample size: } \\
20 \text { patients } \\
10 \text { control }\end{array}$ & $\begin{array}{l}\text { BM-MSC } \\
5 \times 105 / \mathrm{kg}\end{array}$ & $\begin{array}{l}\text { Intralesional followed by } \\
3 \text { monthly intrathecal } \\
\text { injections }\end{array}$ & $\begin{array}{l}\text { Chronic spinal } \\
\text { cord injury }\end{array}$ & $\begin{array}{l}\text { Clinical } \\
\text { ASIA score } \\
\text { MRI }\end{array}$ & $\begin{array}{l}\text { Improvement } \\
\text { Motor and } \\
\text { sensory in } 30 \%\end{array}$ \\
\hline $\begin{array}{l}\text { Kumar et al, } \\
2009(47)\end{array}$ & $\begin{array}{l}\text { Non } \\
\text { randomized } \\
\text { noncontrolledcli } \\
\text { nical trial phase } \\
\text { I/II } \\
\text { Sample size: } \\
297\end{array}$ & $\begin{array}{l}\text { unmanipulated bone } \\
\text { marrow mononuclear } \\
\text { cells }\end{array}$ & lumbar puncture & $\begin{array}{l}\text { Acute and chronic } \\
\text { SCI }\end{array}$ & ASIA protocol & $\begin{array}{l}\text { Sensory and } \\
\text { motor } \\
\text { Improvement in } \\
32.6 \%\end{array}$ \\
\hline $\begin{array}{l}\text { Pal et al } \\
2009(48)\end{array}$ & $\begin{array}{l}\text { Clinical pilot } \\
\text { trial, } \\
\text { uncontrolled } \\
\text { Sample size: } 30 \\
\text { patients }\end{array}$ & $\begin{array}{l}\text { bone marrow }(\mathrm{BM}) \\
\text { mesenchymal stromal } \\
\text { cells }(\mathrm{MSC}) \text {. expanded } \\
\text { ex-vivo } \\
\text { dose: } \\
1 \times 106 \text { cells } / \mathrm{kg}\end{array}$ & lumbar puncture & $\begin{array}{l}\text { Thoracic spinal } \\
\text { cord injury }\end{array}$ & ASIA & Safety \\
\hline Cristante et al, & Prospective & PB leukapharesis- & injected via arteriography & Complete cervical & SSEP & Safety \\
\hline
\end{tabular}




\begin{tabular}{|c|c|c|c|c|c|c|}
\hline Investigators & Study Design & Cell dose \& Type & Mode Of Delivery & Patient diagnosis & Evaluation Scales & Main Results \\
\hline $2009(38)$ & $\begin{array}{l}\text { non-randomized } \\
\text { clinical trial } \\
\text { Sample size: } \\
39 \text { patients }\end{array}$ & $\begin{array}{l}\text { derived mononuclear } \\
\text { cells }\end{array}$ & & and thoracic & & $\begin{array}{l}\text { Improved SSEP } \\
\text { sa }\end{array}$ \\
\hline $\begin{array}{l}\text { Mackay-Sim et } \\
\text { al, } 2008 \text { (49) }\end{array}$ & $\begin{array}{l}\text { Phase I/II } \\
\text { Sample size: } \\
6 \text { patients }\end{array}$ & OEC & Surgical implantation & Complete thoracic & $\begin{array}{l}\text { 1. ASIA } \\
\text { 2. Functional } \\
\text { Indicator measure } \\
\text { assessment } \\
\text { 3. SSEP } \\
\text { 4. MRI }\end{array}$ & $\begin{array}{l}\text { one pafety, } \\
\text { one patient } \\
\text { showed } \\
\text { improvement } \\
\text { in } \\
\text { improvet } \\
\text { w }\end{array}$ \\
\hline $\begin{array}{l}\text { Subbaiah et al, } \\
2008(50)\end{array}$ & $\begin{array}{l}\text { Pilot clinical } \\
\text { trial } \\
\text { Sample size: } \\
5 \text { patients }\end{array}$ & $\begin{array}{l}\text { BM-MSCs stimulated } \\
\text { with brain neutrophic } \\
\text { factor }\end{array}$ & Intrathecal & $\begin{array}{l}\text { Chronic spinal } \\
\text { cord injury }\end{array}$ & ASIA score & $\begin{array}{l}2 \text { patients } \\
\text { improved }\end{array}$ \\
\hline $\begin{array}{l}\text { Chernykh et al, } \\
2007 \text { (37) }\end{array}$ & $\begin{array}{l}\text { Controlled } \\
\text { clinical trial }\end{array}$ & $\begin{array}{l}\text { BMMNCs into cyst } \\
\text { and intravenous }\end{array}$ & $\begin{array}{l}\text { Surgical meningomyelo } \\
\text { radiculosis and } \\
\text { transplatation of cells into } \\
\text { cyst and intravenous }\end{array}$ & Chronic & $\begin{array}{l}\text { ASIA } \\
\text { Bartel } \\
\text { Ashworth }\end{array}$ & $\begin{array}{l}\text { Safey and } \\
\text { motor } \\
\text { improvement }\end{array}$ \\
\hline $\begin{array}{l}\text { Yoon et al, } 2007 \\
(36)\end{array}$ & $\begin{array}{l}\text { Phase I/II } \\
\text { Open label } \\
\text { Non- } \\
\text { randomized } \\
\text { Controlled } \\
\text { Sample size: } \\
35 \text { patients }\end{array}$ & $\begin{array}{l}\text { BM-MNC } \\
+ \\
\text { GCSF }\end{array}$ & Intrathecal & $\begin{array}{l}\text { Acute and Chronic } \\
\text { spinal cord injury }\end{array}$ & $\begin{array}{l}\text { AIS scale } \\
\text { Electrophysiology } \\
\text { MRI }\end{array}$ & $\begin{array}{l}\text { Improvement in } \\
\text { acute, not } \\
\text { chronic } \\
\text { Increased } \\
\text { neuropathic } \\
\text { Pain }\end{array}$ \\
\hline $\begin{array}{l}\text { Lima et al, } 2006 \\
\text { (35) }\end{array}$ & $\begin{array}{l}\text { Pilot clinical } \\
\text { trial } \\
\text { Sample size: } 6 \\
\text { patients }\end{array}$ & OEC & Surgical Implantation & $\begin{array}{l}\text { Chronic spinal } \\
\text { cord injury }\end{array}$ & ASIA Scale & $\begin{array}{l}2 \text { patients } \\
\text { showed } \\
\text { improved } \\
\text { Sensory and } \\
\text { sphincteric } \\
\text { Function } \\
\text { Improved ASIA } \\
\text { score in } \\
\text { All patients }\end{array}$ \\
\hline $\begin{array}{l}\text { Moviglia et al, } \\
2006(51)\end{array}$ & $\begin{array}{l}\text { Pilot clinical } \\
\text { trial } \\
\text { Sample size: } \\
2 \text { patients }\end{array}$ & $\begin{array}{l}\text { MSCS CO- } \\
\text { CULTURED with T } \\
\text { cells and induced to } \\
\text { form neural SC } \\
\text { Dose: } 5 \text { X106-1 X109 } \\
\text { T cells }\end{array}$ & Intra-arterial & $\begin{array}{l}\text { Chronic spinal } \\
\text { cord injury }\end{array}$ & & \\
\hline $\begin{array}{l}\text { Callera and do } \\
\text { Noscimento, } \\
2006 \text { (52) }\end{array}$ & $\begin{array}{l}\text { Pilot clinical } \\
\text { trial }\end{array}$ & $\begin{array}{l}\text { Autologous BM } \\
\text { precursor cells }\end{array}$ & $\begin{array}{l}\text { Injected via lumbar } \\
\text { puncture }\end{array}$ & $\begin{array}{l}\text { Chronic spinal } \\
\text { cord injury }\end{array}$ & & Safety سسس sam \\
\hline $\begin{array}{l}\text { Feron et al, } \\
2005(34)\end{array}$ & $\begin{array}{l}\text { Phase I, } \\
\text { controlled, } \\
\text { single -blind } \\
\text { Sample size: } \\
3 \text { patients } \\
3 \text { control }\end{array}$ & $\begin{array}{l}\text { OEC } \\
\text { Dose: } \\
12 \text { X106 } \\
24 \times 106 \\
48 \times 106\end{array}$ & $\begin{array}{l}\text { Direct injection into the } \\
\text { spinal cord under } \\
\text { microscopic control }\end{array}$ & $\begin{array}{l}\text { Chronic spinal } \\
\text { cord injury }\end{array}$ & $\begin{array}{l}\text { 1. ASIA scale } \\
\text { 2. Mental health } \\
\text { assessment } \\
\text { 3. Clinical outcome } \\
\text { variable (COV) scale } \\
\text { for respiratory } \\
\text { functions }\end{array}$ & $\begin{array}{l}\text { 1. Safety } \\
\text { 2. Minimal } \\
\text { Improvement in } \\
\text { Light touch and } \\
\text { Pinprick } \\
\text { 3. No motor } \\
\text { improvement, } \\
\text { 2. }\end{array}$ \\
\hline
\end{tabular}

\section{Technical Considerations}

Analysis of data gathered from the previously mentioned trials answers a number of questions and raises other problematic issues. The collective patient population covered in trials thus far is 976 spinal cord injury patients. This is an adequate sample to draw some conclusions; with careful consideration of the differences in trial settings. 


\subsection{Type of Study}

As regards the type of the study, only four of the published trials were controlled, only two of them were randomized. The other studies were all uncontrolled pilot clinical trials, apart from one case-control series (41).

\subsection{Patient Choice}

Most trials focused on complete chronic SCI, Kumar et al (47) enrolled both acute and chronic SCI. Moreover, most trials enrolled SCI without subclassification according to injury level. Cristante et al (38) compared cervical and thoracic SCI and Gabr et al (46) compared cervical, thoracic and lumbar lesions.

\subsection{Stem Cell Type}

The earliest trials used olfactory ensheathing cells as a graft in the injured area of the cord. Although the procedure showed some success, it was an extremely invasive procedure both in obtaining the cells and in transplantation. The minimal improvement did not justify the lengthy and complicated procedure $(34,35,49)$.

The next trend used unmanipulated bone marrow mononuclear cells Karamouzian et al (44), Kumar et al (47), ,
Chernykh et al (37), Yoon et al (36), and Callera et al (52); while Cristante et al (38) used leukapharesis derived mononuclear cells. These studies reported variable improvement and short term safety. However, longer safety studies are essential. The possibility of development of autoimmune reaction or sterile inflammatory reaction due to the injection of immunocompetent cells has to be ruled out.

MSCs were introduced as an ideal cell for regenerative medicine, with the advantages of easy isolation, propagation and expansion. Moreover, they were proven to have neuroplasticity, immunomodulatory and positive paracrine action promoting neuroregeneration, Dai et al (43), Pal et al (48), Gabr et al (46), El Kheir et al $(39,42)$, Kishk et al (41) and Abdelaziz et al (40) used bone marrow derived MSCs. Elkheir et al 2010 compared bone marrow and leukapharesis derived MSCs (39).

To augment the neuroregenerative effect of transplanted MSCs, ex-vivo manipulation can be induced. Pal et al (48) and Park et al (45) used ex-vivo expanded MSCs, Moviglia et al (51) used MSCs induced to transdifferentiate into neural stem cells. Subbaiah et al (50) used brain neurotrophic factor to induce neural differentiation in MSCs.

None of the listed studies compared between unmanipulated and manipulated MSCs.

Table (2). Comparison between various cell types used in clinical trials.

\begin{tabular}{|c|c|c|c|}
\hline Cells type & Advantages & Disadvantages & Studies \\
\hline 1. OEC & Central nervous system tissue & Complex procedure & $\begin{array}{l}\text { Lima et al (35), Feron et al (34),Mackay-Sim et al } \\
\text { (49) }\end{array}$ \\
\hline 2. $\mathrm{MNC}$ & Easy isolation & $\begin{array}{l}\text { Mixed population } \\
\text { Immunocompetent and inflammatory } \\
\text { cell contamination }\end{array}$ & $\begin{array}{l}\text { Karamouzian et al (44), } \\
\text { Kumar et al (47), Chernykh et al (37), Yoon et al (36), } \\
\text { Callera et al } \\
\text { (52). }\end{array}$ \\
\hline 3. MSC & Easy isolation, easy expansion & Very small numbers & $\begin{array}{l}\text { Dai et al,(43) Gabr et al, (46),Elkheir et al }(39,42) \text {, } \\
\text { Kishk et al (41), Abdelaziz et al }(40)\end{array}$ \\
\hline $\begin{array}{l}\text { 4. Ex-vivo } \\
\text { manipulated MSC }\end{array}$ & Large number, neural induction & $\begin{array}{l}\text { Possiblity of contamination and gene } \\
\text { mutation }\end{array}$ & $\begin{array}{l}\text { Pal et al (48), Moviglia et al (51), Park et al (45), } \\
\text { Subbaiah et al (50) }\end{array}$ \\
\hline
\end{tabular}

\subsection{Route of Injection}

The main routes of stem cell transplantation were surgical implantation, intrathecal injection and systemic intra-arterial injection. Most studies used the intrathecal injection, with reported positive results. The studies using OEC used open surgical intervention Lima et al (35), Mackay-Sim et al (49); while Feron et al (34) used OEC as a cellular graft injected directly into the spinal cord under microscopic guidance . However MSC or MNC implantation using surgical procedure did not report superior results justifying the invasive procedure Abdelaziz et al (40), Chernyck et al (37), Park et al (45). The use of intra-arterial route is limited due to the technical difficulty.

Table 3. Comparison Between Routes of Stem Cell Injection

\begin{tabular}{lll}
\hline Route & Advantages & Disadvantages \\
\hline 1. Intrathecal & Technically easy & ?BBB, cell loss \\
2. Intravenous & Cross BBB??? & Technical difficulty \\
3. Intra-spinal & Prevention of cell loss & Invasive procedure \\
\hline
\end{tabular}

\subsection{Cell Dose}

Although this is one of the most important issues in stem cell therapy, none of the published clinical trials performed dose escalation study. Cell "dose" estimation is not applicable in OEC grafts as it is not handled as a cellular graft in the studies of Lima et al (35) and Mackay-Sim et al (49). However, Feron et al (34) used celullar OEC. This is the only dose escalation study done, and the reported results showed no correlation between the cell dose and the clinical efficacy. The cell dose used in the published studies varied according to the cell type used. Intrathecally-injected MSCs varied from $1 \times 10^{6} / \mathrm{kg}$ used by Pal et al (48) to $4 \times 10^{7}$ used by Park et al (45).

\subsection{Method of Evaluation}

Evaluation of the efficacy of stem cell therapy is a critical paramter in determining the feasibility of the procedure as well as in comparing variables in cell type, dose, injection regimen and route of transplantation. Objective evaluation 
methods are essential to give evidence-based results that can influence the decision making for patients in the future. Clinical evaluation was used by all studies, ranging from disability scales (ASIA scale, AIS scale, Ashworth scale and functional independence measure) to scores such as ASIA score. The importance of these clinical scoring systems is that they reflect the change in quality of life of the patients as a result of improving their independence. This is the most important aspect of therapy. However, the need for biologic and scientific evidence is still needed. Some trials used MRI and SSEP to evaluate the regeneration at the anatomic and functional levels.

In order to design an objective evaluation measure, the mechanism of regenerative action should be known. Up to this point in research, none of the postulated mechanisms of actions has been proven; which make us far from reaching one objective evaluation measure. Until this is finalized, assessment addressing the clinical efficacy is the most acceptable.

\subsection{Complications}

Apart from self-limited complications related to cell infusion, none of the published clinical trials reported complications due to stem cell therapy. However, longer follow-up is needed to confirm safety of the procedure.

\section{Conclusions}

After a decade of clinical trials involving stem cell therapy for traumatic spinal cord injury, we can say, on solid ground, that regeneration can occur after spinal cord injuries. Clinical trials thus far all documented short term safety and limited efficacy. However, the two important shortcomings are: study design and biologic or physiologic explanations. Challenges for future research are to augment the regenerative effect and verify the exact physiologic mechanism of repair. To reach this, verification of cell type, dose, cell tracking are needed. In addition, construction of randomized controlled trials is essential.

\section{References}

[1] Furlan JC, Sakakibara BM, Miller WC, Krassioukov AV (2013): Global incidence and prevalence of traumatic spinal cord injury. Can J Neurol Sci.;40(4):456-64.

[2] Lakshmipathy U, Verfaillie C (2005): Stem Cell Plasticity. Blood Rev 19(1):29-38.

[3] Woodburry D, Schwarz EJ, Prockop DJ (2000): Adult rat and human bone marrow stromal cells differentiate into neurons. J Neurosc Res 61(4):364-370.

[4] Zhang SCm Wermig M, Duncan ID Brustle O (2001): In vitro differentiation of transplantable neural precursors from human embryonic stem cells. Nature Biotech 19:1129-1133.

[5] Sanchez-Ramos J, Song S, Cardozo=Pelaez F, Hazzi C, Stedeford T, Willing A, Freeman TB, Saporta S, Janssen W, Patel N, Cooper DR, Sanberg PR (2000): Adult Bone Marrow
Stromal Cells Differentiate into Neural Cells in Vitro. Exp Neurol 164(2):247-256.

[6] Mostafavi FS, Razavi S, Mardani M, Esfandiari E, Esfahani HZ and Kazemi M.(2014): Comparative Study of Microtubule-associated Protein-2 and Glial Fibrillary Acidic Proteins during Neural Induction of Human Bone Marrow Mesenchymal Stem Cells and Adipose-Derived Stem Cells. Int J Prev Med.; 5:584-95.

[7] da Silva Meirelles L, Chagastelles PC and Nardi NB.(2006): Mesenchymal stem cells reside in virtually all post-natal organs and tissues. J Cell Sci.; 119:2204-13.

[8] Dezawa M, Takahashi I, Esaki M, Takano M and Sawada H. (2001): Sciatic nerve regeneration in rats induced by transplantation of in vitro differentiated bone-marrow stromal cells. Eur J Neurosci.; 14:1771-6.

[9] Kalbermatten DF, Schaakxs D, Kingham PJ and Wiberg M. (2011): Neurotrophic activity of human adipose stem cells isolated from deep and superficial layers of abdominal fat. Cell Tissue Res.; 344:251-60.

[10] Razavi S, Razavi MR, Zarkesh Esfahani H, Kazemi M and Mostafavi FS.(2013): Comparing brain-derived neurotrophic factor and ciliary neurotrophic factor secretion of induced neurotrophic factor secreting cells from human adipose and bone marrow-derived stem cells. Dev Growth Differ.; 55:64855 .

[11] Liao D, Gong P, Li X, Tan Z and Yuan Q.(2010) Co-culture with Schwann cells is an effective way for adipose-derived stem cells neural transdifferentiation. Arch Med Sci.; 6:145-51.

[12] Zhang WC, Ding YJ, Cao JK, Du JX, Zhang GF and Liu YJ.(1994): Intracerebral cografting of Schwann's cells and fetal adrenal medulla in the treatment of Parkinson's disease. Chin Med J (Engl); 107:583-8.

[13] Jiang CC, Xia Y, Ding ZL, Wang Y and Xu B.(2006): Effects of co-engraftment of Schwann cells with neural stem cells into rats with Parkinson disease. Chin Med J (Engl). ; 119:1030-3.

[14] Sadan O, Shemesh N, Barzilay R, Bahat-Stromza M, Melamed E, Cohen Y and Offen D. (2008): Migration of neurotrophic factors-secreting mesenchymal stem cells toward a quinolinic acid lesion as viewed by magnetic resonance imaging. Stem Cells.; 26:2542-51.

[15] Crigler L, Robey RC, Asawachaicharn A (2006):. Human mesenchymal stem cell subpopulations express a variety of neuroregulatory molecules and promote neuronal cell survival and neuritogenesis. Exp Neurol.;198:54-64.

[16] Ma T, Gong K, Ao Q, Yan Y, Song B, Huang H, Zhang X and Gong Y. (2013): Intracerebral transplantation of adiposederived mesenchymal stem cells alternatively activates microglia and ameliorates neuropathological deficits in Alzheimer's disease mice. Cell Transplant.; 22 Suppl 1:S11326.

[17] Hu BYm Weick JPm Yu J, Ma L, Zhang Z, Thomson J and Zhang S (2010): Neural differentiation of human induced pluripotent stem cells follows developmental principles but with variable potency. PNAS 107(9).

[18] Swistowski A1, Peng J, Liu Q, Mali P, Rao MS, Cheng L, Zeng X.(2010): Efficient generation of functional dopaminergic neurons from human induced pluripotent stem cells under defined conditions. . Stem Cells. 2010 Oct;28(10):1893-904. 
[19] Paldino E1, Cenciarelli C, Giampaolo A, Milazzo L, Pescatori M, Hassan HJ, Casalbore P.(2014): Induction of dopaminergic neurons from human Wharton's jelly mesenchymal stem cell by forskolin J Cell Physiol. 2014 Feb;229(2):232-44.

[20] Wichterle H1, Lieberam I, Porter JA, Jessell TM.(2002): Directed differentiation of embryonic stem cells into motor neurons. Cell. 2002 Aug 9;110(3):385-97.

[21] Selvaraj V1, Jiang P, Chechneva O, Lo UG, Deng W. (2012): Differentiating human stem cells into neurons and glial cells for neural repair.Front Biosci (Landmark Ed). 2012 Jan $1 ; 17: 65-89$

[22] Hardy SA, Maltman DJ, Przyborski SA. (2008): Mesenchymal stem cells as mediators of neural differentiation.Curr Stem Cell Res Ther. ;3:43-52.

[23] Madrigal M, Rao KS, Riordan NH (2014): A review of therapeutic effects of mesenchymal stem cell secretions and induction of secretory modification by different culture methods. J Transl Med 12:260.

[24] Razavi S, Jahromi M, Amirpour N and Khosravizadeh Z. Effect of sertraline on proliferation and neurogenic differentiation of human adipose-derived stem cells (2014): Adv Biomed Res.; 25:97.

[25] Radtke C, Schmitz B, Spies M, Kocsis JD and Vogt PM.(2009): Peripheral glial cell differentiation from neurospheres derived from adipose mesenchymal stem cells. Int J Dev Neurosci.; 27:817-23.

[26] Gebler A, Zabel O, Seliger B (2012): The immunomodulatory capacity of mesenchymal stem cells. Trends in Mol Med 18(2):p128-134.

[27] Hoogduijn MJ, Popp F, Verbeek R, Masoodi M , Nicolaou A, Baan , Dahlke M (2010): The immunomodulatory properties of mesenchymal stem cells and their use for immunotherapy. Int Immunopharmacolog 10(12):1496-1500.

[28] Huang WH, Chang MC, Tsai KS, Hung MC, Chen HL, Hung SC. (2013): Mesenchymal stem cells promote growth and angiogenesis of tumors in mice. . Oncogene. ;32(37):4343-54.

[29] Martins L, Martin PK, Han SW. (2014): Angiogenic properties of mesenchymal stem cells in a mouse model of limb ischemia. Methods Mol Biol.;1213:147-69

[30] Joyce N, Annett G., Wirthlin L, Olson S, Bauer G, and Nolta JA (2010): Mesenchymal stem cells for the treatment of neurodegenerative disease Regen Med.; 5(6): 933-946.

[31] Kramer R, Zhang Y, Gehrmann J, Gold R, Thoenen H and Wekerle H. (1995): Gene transfer through the blood-nerve barrier: NGF-engineered neuritogenic $\mathrm{T}$ lymphocytes attenuate experimental autoimmune neuritis. Nat Med. ; 1:1162-6.

[32] Kim JH, Lee HJ, and Song JS (2014): Stem Cell Based Gene Therapy in Prostate Cancer

[33] BioMed Res Int; 2014:Article ID 549136, 8 pages. Uchibori R, Tsukahara T, Ohmine K, Ozawa K. (2014): Cancer gene therapy using mesenchymal stem cells. Int J Hematol.;99(4):377-82.

[34] Feron F, Perry C, Cochrane J, Licina P, Nowitzke A, Urquhart S, Geraghty T, Mackay-Sim A (2005): Autologous olfactory ensheathing cell transplantation in human spinal cord injury. Brain 657: 2951-2960.
[35] Lima C, Pratas-Vital J, Escada P, Hasse-Fereira A, Capucho C, Peduzzi JD (2006): Olfactory mucosa autografts in human spinal cord injury: a pilot clinical study. J Spinal Cord Med; 29(3): 191-203.

[36] Yoon SH, Shim YS, Park YH, Chung JK, Nam JH, Kim MO, Park HC, Park SR, Min BH, Kim EY, Choi BH, Park H, Ha Y (2007): Complete spinal cord injury treatment using autologous bone marrow cell transplantation and bone marrow stimulation with granulocyte macrophage-colony stimulating factor: Phase I/II clinical trial. . Stem Cells.25(8):2066-73.

[37] Chernykh ER, Stupak VV, Muradov GM, Sizikov MY, Shevela EY, Leplina OY, Tikhonova MA, Kulagin AD, Lisukov IA, Ostanin AA, Kozlov VA (2007): Application of autologous bone marrow stem cells in the therapy of spinal cord injury patients. Bull Exp Biol Med. ;143(4):543-7.

[38] Cristante AF, Barros-Filho TE, Tatsui N, Mendrone A, Caldas JG, Camargo A, Alexandre A, Teixeira WG, Oliveira RP, Marcon RM (2009): Stem cells in the treatment of chronic spinal cord injury: evaluation of somatosensitive evoked potentials in 39 patients. Spinal Cord. 47(10):733-8.

[39] Abo El-Kheir W, Gabr H, Awad MR, von Wild K, and Ramadan M (2010): Autologous Mesenchymal Stem Cells for Neuro-Regeneration After Traumatic Spinal Cord Injury: A Comparison Between Bone Marrow and Peripheral Blood Populations. Am J Neuroprot and Neuroregene; 2: 1-8.

[40] Abdelaziz OS, Marie A, Abbas M, Ibrahim M, Gabr H (2010): Feasibility, Safety, and Efficacy of Directly Transplanting Autologous Adult Bone Marrow Stem Cells in Patients With Chronic Traumatic Dorsal Cord Injury. A Pilot Clinical Study. Neurosurg Q 2010;20:216-226.

[41] Kishk NA, Gabr H, Hamdy S, Afifi L, Abokresha N, Mahmoud H, Wafaie A and Bilal D ( 2010): Case Control Series of Intrathecal Autologous Bone Marrow Mesenchymal Stem Cell Therapy for Chronic Spinal Cord Injury. Neurorehabil Neural Repair.

[42] El-Kheir WA, Gabr H, Awad MR, Ghannam O, Barakat Y, Farghali HA, Maadawi ZM, Ewes I, Sabaawy HE. (2013):Autologous bone marrow-derived cell therapy combined with physical therapy induces functional improvement in chronic spinal cord injury patients. Cell Transplant.

[43] Dai G, Liu $X$, Zhang $Z$, Yang Z, Dai $Y$, Xu R (2013): Transplantation of autologous bone marrow mesenchymal stem cells in the treatment of complete and chronic cervical spinal cord injury. Brain Res.;1533:73-9.

[44] Karamouzian S, Nematollahi-Mahani SN, Nakhaee N, Eskandary H.(2012): Clinical safety and primary efficacy of bone marrow mesenchymal cell transplantation in subacute spinal cord injured patients. Clin Neurol Neurosurg.;114(7):935-9.

[45] Park JH, Kim DY, Sung IY, Choi GH, Jeon MH, Kim,K, Jeon SR 2012): ): Long-term Results of Spinal Cord Injury Therapy Using Mesenchymal Stem Cells Derived From Bone Marrow in Humans. Neurosurgery: 70(5):1238-1247.

[46] Gabr, H.; Ghannam, O.; Awad, MR; von Wild, K.; El-Kheir, W.; Ewes, I. (2011): Autologous Mesenchymal Stem Cell Therapy for Spinal Cord Injury: Long Term Safety and Clinical Efficacy American Journal of Neuroprotection and Neuroregeneration, 3( 1):100-106(7). 
[47] Kumar AA, Kumar SR, Narayanan R, Arul K, Baskaran M.(2009): Autologous bone marrow derived mononuclear cell therapy for spinal cord injury: A phase I/II clinical safety and primary efficacy data. Exp Clin Transplant.;7(4):241-8.

[48] Pal R, Venkataramana NK, Bansal A, Balaraju S, Jan M, Chandra R, Dixit A, Rauthan A, Murgod U, Totey S (2009): Ex vivo-expanded autologous bone marrow-derived mesenchymal stromal cells in human spinal cord injury/paraplegia: a pilot clinical study. Cytotherapy.;11(7):897-911.

[49] Mackay-Sim A, Féron F, Cochrane J, Bassingthwaighte L, Bayliss C, Davies W, Fronek P, Gray C, Kerr G, Licina P, Nowitzke A, Perry C, Silburn PA, Urquhart S, Geraghty T.(2008): Autologous olfactory ensheathing cell transplantation in human paraplegia: a 3-year clinical trial. Brain.;131(Pt 9):2376-86.
[50] Subbaiah G, Adavi V, Chelluri L, Laxman S, Ratnakar K, Gopal P, Ravindranath K(2008):Preliminary report on the safety, efficacy and functional recovery of spinal cord injury with autologous bone marrow derived mesenchymal stem cells - a clinical trial. Int J Spine Surg 5(1).

[51] Moviglia GA, Fernandez Viña R, Brizuela JA, Saslavsky J, Vrsalovic F, Varela G, Bastos F, Farina P, Etchegaray G, Barbieri M, Martinez G, Picasso F, Schmidt Y, Brizuela P, Gaeta CA, Costanzo H, Moviglia Brandolino MT, Merino S, Pes ME, Veloso MJ, Rugilo C, Tamer I, Shuster GS.(2006): Combined protocol of cell therapy for chronic spinal cord injury. Report on the electrical and functional recovery of two patients. Cytotherapy; 8(3):202-9.

[52] Callera F, do Nascimento RX. (2006): Delivery of autologous bone marrow precursor cells into the spinal cord via lumbar puncture technique in patients with spinal cord injury: a preliminary safety study. Exp Hematol. ;34(2):130-1. 